\title{
Influence of pre-treatment on torrefaction of Phyllostachys edulis
}

\author{
Xiwei $\mathrm{Xu}^{1,2}$ Enchen Jiang ${ }^{1} *$ Xiang $\operatorname{Lan}^{1}$ \\ 1. College of Materials and Energy, South China Agricultural University, Guangzhou 510640 \\ 2. Key Laboratory of Biomass Energy and Material, Jiangsu Province, Nanjing 210007
}

\begin{abstract}
This study investigated the effects of different pre-treatments on structural changes in Phyllostachys edulis. Samples were pretreated with water, 15\% ammonia water, 2\% sulfuric acid, hydrothermal carbonization, and ball milling. Moreover, ultrasound was introduced. The influence of pre-treatment on the physiochemical property and composition of $P$. edulis were studied. Moreover, torrefaction characterization was performed and the distribution of torrefaction products of pretreated samples was determined. Results showed that pre-treatment effectively modified physiochemical structure and the torrefaction property of $P$. edulis. The pretreatment reduced the ash content and increased the bio-oil content of the torrefaction products. Compared with that of the raw material, the residual bio-char content of the pretreated samples decreased by $2 \%-8 \%$, and degradation temperature of bio-char fluctuated between $365^{\circ} \mathrm{C}$ and $321^{\circ} \mathrm{C}$. The distribution of bio-oil contents, bio-char, and bio-gas in the torrefaction products significantly varied with pretreatments methods.
\end{abstract}

Keywords: Phyllostachys edulis; pre-treatment; torrefaction property, product distribution.

\section{Introduction}

Lignocellulosics are widely found in plants, and they display worldwide distribution. And lignocellulosics is considered as the promising renewable materials and are also widely used to derive renewable and clean energy through chemical conversion and bioconversion.

The traditional biomass is used to produce bio-oil, ethanol, or bio-gas by bioconversion. However, the polymeric lignocellulosics cannot be directly fermented 
to produce bio-material. Thus, a pretreatment is necessary and essential. In the past decades, researchers had focused on the effect of pre-treatment on bioconversion of lignocellulosics into liquid and gas products (Chen et al., 2016; Aidet et al., 2016; Carvalho et al., 2016; Si et al., 2015; Agbor et al., 2011). Pretreatment mainly disrupts the chemical structure of lignocellulose, reduces the relative crystallinity of cellulose, and increases the ratio of surface area and pore volume to pore diameter of cellulose, among others. Moreover, a pre-treatment changes the chemical structure, controls the product distribution, and improves the yield and quality of certain substances during thermo-chemical conversion of biomass. Therefore, the influences of pretreatment on the internal structure of biomass and on the characteristics of pyrolysis products present important practical significance and commercial value. For example, Raveendran et al. (2017) developed the ultrasound-assisted alkali pretreatment strategy, which effectively removed lignin and hemicelluloses as well as the major fermentation inhibitors. Karray et.al. (2015) found that ultrasound improved pre-treatment methods, reduced pre-treatment time, increased yields and brought down moderate costs and negligible toxicity added. Ultrasound was used for the disruption of cell of biomass. Lu et al. (2017) investigated the influence of hydrothermal pretreatment conditions on ethanol production. However, the influence of pretreatment on chemical conversion, such as pyrolysis and catalytic reforming, is rarely reported.

Pyrolysis of biomass is an effective method for biomass conversion for producing renewable energy. Torrefaction is widely used as one of pretreatment methods for the pyrolysis or catalytic reforming of biomass. Torrefaction, which was an effective route to enhance the yield and heat value of bio-char, to reduce the oxygen content of bio-oil, and to alter the composition of biogas ( Woytiuk et al., 2017; Doddapaneni et al., 2016; Wigley et al., 2016; Chen et al., 2017 ), became controversial in recent years. Researchers had found that torrefaction affected biomass properties, such as moisture content, calorific value, increased heating value, energy yield, atomic H/C, O/C ratios, grindability, and biodegradability (Tran et al., 2014; Basu et al., 2014). For example, Wigley et al. (2016) had developed a pre-treatment 
sequence incorporating acid leaching and torrefaction to reduce/remove the catalytic role of inorganics, acids, and water. Chen et al. (2015) suggested that optimization of torrefaction operation depended on energy densification or energy utilization. Moreover, Zhang et al. (2016) investigated the influence of wet torrefaction on the physicochemical properties and combustion behavior of duckweed biomass at $130{ }^{\circ} \mathrm{C}-250{ }^{\circ} \mathrm{C}$.

This study investigated the influence of pre-treatment of biomass on products distribution and characteristics of torrefaction products.

\section{Materials and methods}

\subsection{The raw materials}

Phyllostachys edulis collected from Anji, Zhejiang Province was used as test material. Samples were crushed by a YB-1000A pulverizer (Yongkang Sufeng Industry and Trade Co., Ltd.). Raw materials were screened by 30-, 80-, and 200-mesh screens and then dried at $50^{\circ} \mathrm{C}$ for $24 \mathrm{~h}$.

\subsubsection{Methods for sample pre-treatment}

Pre-treatment with water: a certain mass of dry raw material screened and go through \#30 mesh was collected and immersed in a thermostatic water in a thermostatic water bath containing distilled water after distilled water was added. The specific pre-treatment conditions were as follows: $1 \mathrm{~g}$ : $5 \mathrm{~mL}$ solid-liquid mass-to-volume ratio, $90{ }^{\circ} \mathrm{C}$ reaction temperature, and $12 \mathrm{~h}$ pre-treatment. After the reaction was completed, the samples were filtered by a SHB-III circulating water vacuum pump (Shanghai Yuming Instrument Co., Ltd.) and washed with distilled water until the $\mathrm{pH}$ is 7 . All solid products were dried overnight at $50{ }^{\circ} \mathrm{C}$; the washed pre-treated samples were marked as zzsx.

Pre-treatment with ammonia solution: a certain mass of dry raw material screened by 30-mesh was collected and immersed in a thermostatic water bath after ammonia water was added. The specific pre-treatment conditions were as follows: 15\% (mass fraction) ammonia water, $1 \mathrm{~g}: 5 \mathrm{~mL}$ solid-liquid mass-to-volume ratio, $60{ }^{\circ} \mathrm{C}$ reaction temperature, and $12 \mathrm{~h}$ pre-treatment. After the reaction was completed, the samples were filtered under vacuum conditions and washed with distilled water until neutral. All solid products were dried overnight at $50{ }^{\circ} \mathrm{C}$; the samples pre-treated 
with ammonia water were marked as zzas.

Ultrasonic pre-treatment with ammonia solution was performed at a power of $300 \mathrm{~W}$ and a frequency of $28 \mathrm{kHz}$ in aqueous soda solution. The pre-treatment conditions were similar to those in the pre-treatment with ammonia solution. The samples pre-treated with ammonia water were marked as zzcas.

Ultrasonic pre-treatment with sulfuric acid solution: a certain mass of dry raw material screened by 30 -mesh was collected and immersed in a thermostatic water bath under ultrasonic wave under a power of 300W and a frequency of $28 \mathrm{kHz}$ after sulfuric acid was added. The specific pre-treatment conditions were as follows: $2 \%$ (mass fraction) sulfuric acid, $1 \mathrm{~g}: 5 \mathrm{~mL}$ solid-liquid mass-to-volume ratio, $60{ }^{\circ} \mathrm{C}$ reaction temperature, and 30 min pre-treatment. After the reaction was completed, the samples were filtered under vacuum conditions and thoroughly washed with distilled water until neutral. All solid products were dried overnight at $50{ }^{\circ} \mathrm{C}$; the samples pre-treated with ultrasonic sulfuric were marked as zzcls.

Pre-treatment with sulfuric acid solution: a certain mass of dry raw materials screened by different meshes were collected and immersed in a thermostatic water bath after sulfuric acid was added. The pre-treatment conditions were as follows: $2 \%$ (mass fraction) sulfuric acid, $1 \mathrm{~g}: 5 \mathrm{~mL}$ solid-liquid mass-to-volume ratio, $90{ }^{\circ} \mathrm{C}$ reaction temperature, and $12 \mathrm{~h}$ pre-treatment. After the reaction was completed, the samples were filtered under vacuum conditions and thoroughly washed with distilled water until neutral. All solid products were dried overnight at $50{ }^{\circ} \mathrm{C}$; samples pre-treated with sulfuric acid and screened under different meshes were finally obtained. The samples were marked as zz30ls, zz80ls, and zz200ls according to particle size.

Pre-treatment with hydrothermal carbon: approximately $5.0 \mathrm{~g}$ of dry raw materials screened by 30-mesh were collected and added into $100 \mathrm{~mL}$ hydrothermal reactor. Distilled water was added based on the following proportion: 1 part raw material: 5 parts distilled water. The reaction temperature increased from room temperature to $180{ }^{\circ} \mathrm{C}$ and stabilized at this temperature for $4 \mathrm{~h}$. The mixture was kept at room temperature, filtered under vacuum conditions, and thoroughly washed with distilled water until neutral. All solid products were dried overnight at $50{ }^{\circ} \mathrm{C}$; the samples pre-treated with hydrothermal carbon were marked as zzsr.

Pre-treatment with Ball mill grinding: the raw material was ground into micro 
scale by using a PM Series Planetary Ball Mill (Nanjing Chishun Science and Technology Development Limited Company). Each tank contained $1.5 \mathrm{~g}$ of dry raw material screened by 80 -mesh and ground by balls at a rate of $200 \mathrm{r} / \mathrm{min}$. In the ball-milling treatment, the raw material was ground for 6 min and then stopped for 6 min. The ball-milling pre-treatment lasted for $8 \mathrm{~h}$, and pre-treated samples were marked as zzqm.

\subsubsection{Test for physical and chemical properties of $\boldsymbol{P}$. edulis}

The physical and chemical properties of the P. edulis samples were determined using an automatic industrial analyzer (Changsha Youxin Instrument Manufacturing Co., Ltd.) with GB/T28731-2012 as reference.

Calorific value analysis was performed using a YX-ZR Skyhawk Automatic Calorimeter (Changsha Youxin Instrument Manufacturing Co., Ltd.).

The cellulose, lignin, and hemicellulose contents of the samples were determined using the Van Soest method.

All measurements were performed in duplicate and the average value is reported.

Thermogravimetric analysis (TGA): approximately $8 \mathrm{mg}$ of untreated and pre-treated $P$. edulis samples were analyzed on a TG-DSC 449c differential thermogravimeter by using $\mathrm{Al}_{2} \mathrm{O}_{3}$ crucible under nitrogen protection and under increasing temperature from room temperature to $800{ }^{\circ} \mathrm{C}$ at a rate of $10{ }^{\circ} \mathrm{C} / \mathrm{min}$. $\mathrm{A}_{2} \mathrm{O}_{3}$ was used as reference compound.

\subsection{The calculation of the heat value of bio-gas}

The heat value of bio-gas was calculated by the equal:

$\mathrm{Q}_{\text {bio-gas }}=\mathrm{W}_{\mathrm{H} 2} * 3044+\mathrm{W}_{\mathrm{CO}} * 3018+\mathrm{W}_{\mathrm{CH} 4} * 9510+\mathrm{W}_{\mathrm{C} 2 \mathrm{H} 4} * 15142+\mathrm{W}_{\mathrm{C} 2 \mathrm{H} 6} * 16792$ (It is acceptable that the heat value of $\mathrm{H}_{2}, \mathrm{CO}, \mathrm{CH}_{4}, \mathrm{C}_{2} \mathrm{H}_{4}$ and $\mathrm{C}_{2} \mathrm{H}_{6}$ is $3044 \mathrm{Kcal} / \mathrm{Nm}^{3}$, $3018 \mathrm{Kcal} / \mathrm{Nm}^{3}, 9510 \mathrm{Kcal} / \mathrm{Nm}^{3}, 15142 \mathrm{Kcal} / \mathrm{Nm}^{3}$ and $16792 \mathrm{Kcal} / \mathrm{Nm}^{3}$, respectively. And $\mathrm{W}_{\mathrm{H} 2}, \mathrm{~W}_{\mathrm{CO}}, \mathrm{W}_{\mathrm{CH} 4}, \mathrm{~W}_{\mathrm{C} 2 \mathrm{H} 4}$ and $\mathrm{W}_{\mathrm{C} 2 \mathrm{H} 6}$ is the relative content of $\mathrm{H}_{2}, \mathrm{CO}, \mathrm{CH}_{4}, \mathrm{C}_{2} \mathrm{H}_{4}$ and $\mathrm{C}_{2} \mathrm{H}_{6}$ in bio-gas, respectively)

\subsection{Pyrolysis experiment}

A pyrolysis experiment was performed using a SK2-2-10H Resistance Furnace (Shanghai Yifeng Electric Furnace Co., Ltd.). Figure 1 shows the experimental apparatus used in pyrolysis.

The experiment was performed as follows (in figure 1): a condenser was supplied with ice water prior to the reaction to increase the condensation effect. 
Approximately $9 \mathrm{~g}$ of $P$. edulis samples were placed into a railboat. The railboat was placed in front of a quartz tube, and a rubber plug was used to check the air impermeability of the experimental apparatus. The flowrate of nitrogen, which was used as carrier gas, was kept at $200 \mathrm{ml} / \mathrm{min}$ throughout the reaction to drive air in the tube and maintain an anaerobic atmosphere. The program temperature controller was turned on to increase the room temperature to $300{ }^{\circ} \mathrm{C}$. After temperature stabilization, the railboat was pushed quickly into the middle of the quartz tube, and the reaction was initiated. The first bag of gas was collected at the first minute and the second bag of gas was collected at the fifth minute. At $30 \mathrm{~min}$, the railboat was pulled back in front of the quartz tube, and the reaction was stopped. After the railboat had cooled down, carbon mass and liquid product mass were determined, and the gas was quantified by subtraction method. All measurements were performed in duplicate and the average value was reported.

\subsection{Pyrolysis product analysis}

2.3.1 Analysis of biomass carbon using the infrared (IR) spectrum test.

In this test, we used a Thermo Nicolet Avatar 360 FT-IR spectrometer (USA). First, test samples were dried for $12 \mathrm{~h}$ at $75^{\circ} \mathrm{C}$. Second, a certain amount of samples and $\mathrm{KBr}$ were ground and pushed by a tablet press at $25 \mathrm{~Pa}$. IR test was performed on a test table, and the scanning range was $4000-400 \mathrm{~cm}^{-1}$. IR transmittance of the samples were recorded, and the IR spectra of the samples were obtained.

\section{Results and analysis}

\subsection{Analysis of the physical and chemical properties of pretreated samples}

The chemical and physical properties of pretreated samples of P. edulis are listed in Table 1.

A biomass contains a certain amount of metal elements apart from carbon, hydrogen, oxygen, and other organics. These metal elements are mainly $\mathrm{Ca}, \mathrm{K}, \mathrm{Na}$, $\mathrm{Mg}$, whereas $\mathrm{Fe}, \mathrm{Mn}$, and $\mathrm{Al}$ exist in low amounts. They generally exist as oxide, silicide, carbonate, sulfate, chloride, and phosphate. Given the diversity of ash composition, the influences of metal elements on pyrolysis of biomass have become very complicated. Different pre-treatments, such as deashing and metal salt treatment (Zhang et al., 2015) have been introduced. Deashing pre-treatment generally involves washing and acid pickling. Washing dissolves metal salts only, which easily dissolve 
in water, but will not affect the other components of a biomass. Acid pickling not only can eliminate most of metal salts in biomass but also influence the structures of the three components of a biomass. The decreasing order of the effects of different pre-treatments on ash content is as follows: $z z s r>$ zzcls $>$ zzsx $>$ zz30ls $>$ zzas $>$ zzcas $>$ zz80ls > zz200ls > zzqm. The hydrothermal pre-treatment showed the highest deashing effect as manifested in the concentration of organic volatile components and in high fixed carbon content; additionally, deashing promoted the highest calorific value. Apart from zzsr, zzcls showed the lowest ash content, indicating that ultrasonic and acid play a role in the removal of metals in biomass. It is possible that acid can promote the decomposition of organic in biomass. Meanwhile, ultrasound can break down the interaction of metal and organic materials, accelerate the decomposition and remove the metal in the biomass. Therefore, the ash in the samples, which is pretreated with ultrasonic and sulfuric acid solution, is lowest among all samples.

Moreover, in zzcas and zzqm, the volatile matter content in the absence of ash and moisture is lower than that in other samples; the combination of ammonia and ultrasonic treatment is possibly beneficial in removal of organics.

Table 2 shows the amounts of the principal components of the samples The results demonstrated that the raw materials contained as high as 51.55\% cellulose and the content of hemicellulose and lignin was similar, approximately 20\%. The variously pretreated samples showed varied compositions. The cellulose, hemicellulose, and lignin contents of the pretreated samples were fluctuated among 47\%-58\%, 8.6\%-21\%, and $19.6 \%-28.9 \%$, respectively. This result indicated that the amounts of the principal components of $P$. edulis could be significantly changed by pretreatment. Therefore, pretreatment is necessary for getting different products from biomass conversion. The hemicellulose content of zzsr significantly decreased from $18.66 \%$ to $8.69 \%$. Some of the hemicelluloses possibly dissolved in hot water during hydrothermal carbonization. Moreover, after being washed with sulfuric acid, the hemicellulose content significantly decreased. For example, the content of the hemicellulose in the sample zz30ls, zz80ls and zz200ls decreased to $12.47 \%, 13.51 \%$ and $10.06 \% \%$, respectively. It indicates that the acid is possibly beneficial for 
hemicellulose conversion. It is acceptable that some of the pre-treatment will remove the quantities of hemicellulose (HC) which usually contributes to the heat value of the biogas produced during torrefaction. Therefore, it is obvious that energy fraction of biog-as produced from biomass after pretreatment will declined (the change of energy fraction will be detailed explained in the next programmer). However, the simplification of bio-oil composition will realize, which is beneficial for the utilization of bio-oil.

Among the pre-treatments, washing rarely influences the principal components and slightly increases the contents of the three principal components. The result was observed because the amount of soluble impurities decreased after washing pretreatment using a neural detergent, whereas the principal components became concentrated. Cellulose content in the sample ammonia pre-treatment increased significantly from 51.55\% to 58.37\%, whereas hemicellulose and lignin slightly decreased due to the dissolution in alkaline solution.

Ultrasound pretreatment increases the exposure of samples to treatment solutions, breaks down the polymerization of organic materials in samples, generates collapses and microchannels on samples, and removes attachments in samples (He et al., 2017). Ultrasonic pretreatment with ammonia promoted dissolution of lignin, therefore, the content of hemicellulose and cellulose increased. It indicates that the pretreatment displays a certain selectivity in terms of dissolving the principal components. Ultrasonic pre-treatment with sulfuric acid slightly influences the principal components. And the particle size plays a significantly role in the principal components. And the content of hemicellulose decreases slightly with the decline of particle size. That is, the content of hemicellulose decreases to $1.57 \%, 13.51 \%$ and $10.06 \%$ corresponding to the samples 30-mesh, 80-mesh and 200-mesh, respectively. It is possible that the contact area between the pretreated samples and sulfuric acid increases with decreasing particle size of the samples. Therefore, the dissolution rate of hemicellulose increases significantly, and the dissolution rate of cellulose further increases. Besides, it is also one of the reason that sulfuric acid easily disrupts the hemicellulose structure.

It indicates that hemicellulose easily dissolves when they are pretreated with 
sulfuric acid and ultrasonic. Additionally, experimental data suggested that increase of lignin content is related to cellulose dissolution. However, the amount of cellulose and lignin increases due to the dissolution of a considerable amount of hemicellulose.

The hydrothermal pre-treatment is accomplished under the subcritical state of water. Under this condition, the amount of hydrogen ions in the water increases and thus the water becomes acidic and can dissolve hemicellulose, resulting in reduction of hemicellulose content from $18.66 \%$ to $8.69 \%$. Ball-milling mechanically grinds the structural components of the raw material, and these components are dissolved with increasing amount of acid detergent. As a result, hemicellulose content increases and the amounts of cellulose with structural damages and lignin decrease.

Figures 2 and 3 show the TG and TGA results of $P$. edulis pretreated with different methods. Biomass pyrolysis is generally divided into three stages. The first stage is the dehydration stage $\left(<240{ }^{\circ} \mathrm{C}\right)$. Given the low moisture contents of the raw materials, all samples showed mass loss in this stage, although the mass is slightly different. The second stage is corresponding to the mass loss caused by pyrolysis of cellulose, hemicellulose, and lignin. The mass loss in the stage is nearly the same for all samples fluctuated among 69\% to 60\%, except for zzyl, which is a bit less than others (in table 3). The third stage is the slow decomposition of residual substances resulting from torrefaction of $P$. edulis. In the stage, the mass loss for zzas is much higher than others samples, about $24 \%$. And the total mass loss for zzas is maximum, about 94\%.

The principal components of biomass are often hypothesized to be decomposed independently under given operation conditions. It is acceptable that the decomposition temperatures of hemicellulose, cellulose, and lignin are $225^{\circ} \mathrm{C}-350{ }^{\circ} \mathrm{C}$, $325^{\circ} \mathrm{C}-375^{\circ} \mathrm{C}$, and $200^{\circ} \mathrm{C}-500{ }^{\circ} \mathrm{C}$, respectively. The difference in the pyrolysis of the three principal components of the biomass is mainly caused by the internal structure and composition of these components. Obviously, the second stage of pyrolysis is different in the TG and DTG curves of pyrolysis. Table 3 shows the characteristic pyrolysis parameters of different pretreated samples. For the raw materials and pretreated samples, the initial pyrolysis temperature of $P$. edulis fluctuated among $257^{\circ} \mathrm{C}$ and $332{ }^{\circ} \mathrm{C}$, and the initial pyrolysis temperature for all samples is as follows: zzqm $<$ zzyl $<$ zzcls $<$ zzcas $<$ zz30ls $<$ zzas $<$ zzsr; the ending 
temperature fluctuated among $349^{\circ} \mathrm{C}$ and $381^{\circ} \mathrm{C}$, and the order of the samples is as follows: zzqm $<$ zzyl $<$ zzas $<$ zzcls $<$ zz30ls $<$ zzcas $<$ zzsr. It indicates that the pyrolysis shifted to the high temperature zone after pretreating, excepting for zzqm.

Moreover, the temperature corresponding to the maximum reaction rate changes significantly for different samples, ranging from $321^{\circ} \mathrm{C}$ to $365^{\circ} \mathrm{C}$. And the order of the samples is as follows: zzqm $<$ zzcls $<$ zzyl $<$ zz30ls $<$ zzcas $<$ zzas $<$ zzsr.

Compared with that in the sample pretreated with ultrasound in distilled water, the maximum mass loss in zzqm and zzcls shifts to the low-temperature region, whereas the mass loss in zz30ls, zzcas, zzas, and zzsr shifts to the high-temperature region. It is possible that the specific surface area was increased samples pretreated by ball mill grinding, and zzcls is beneficial for fluffy of samples structures, as well as for heat transfer.

The content of hemicellulose, cellulose and even lignin in samples of zz30ls, zzcas, zzas, and zzsr decreased, indicating that the pretreatment methods are effective for the changing of three princle compounds. The maximum mass loss rate fluctuates within 8.6-15.7 wt\%/min for different samples, and the order of the samples in terms of mass loss rate is as follows: zzsr > zzas $>$ zz30ls $>$ zzcas $>$ zzcls $>$ zzqm $>$ zzyl. The result reflects that different pre-treatments will influence the pyrolysis behavior of biomass to some extent. For all samples, the total mass loss is higher than $60 \%$, indicating that the samples contains a considerable amount of degradable organic matters. The mass loss rate of pyrolysis of samples enters into the slow stage when the temperature is beyond $400{ }^{\circ} \mathrm{C}$. It indicated that the pyrolysis reaction of organic matters in P. edulis is basically completed. The third stage is that residual lignin and carbon are subsequently degraded as the temperature is beyond $400^{\circ} \mathrm{C}$. Moreover, we indicate that the third stage is the most energy-consuming stage throughout the pyrolysis reaction, and high-temperature pyrolysis improved the cost of resource utilization of $P$. edulis.

In the DTG curve, there is a weak shoulder peak in the zone of 270 to $320^{\circ} \mathrm{C}$ for all pretreated samples. It corresponds to the mass loss due to pyrolysis of hemicellulose. Especially, the peak is obvious for zzas and zzcas. Because ammonia water is beneficial for the degradation of lignin and the destruction of the structure of the protective layer formed from cellulose and hemicellulose. The encapsulated 
hemicellulose is exposed to ammonia water, making it more easily degradable at lower temperature. The shoulder peak has become even more obvious after the combined pre-treatment of ultrasonic wave and ammonia water, and the molecular porosity further increases under the effect of ultrasonic wave. The shoulder peak under the other pre-treatments changes slightly or disappears because the cellulose structure loosens, which is conducive for heat and mass transfers, thereby weakening the pyrolysis line of hemicellulose and cellulose. Hemicellulose and cellulose could not be distinguished clearly in the TG and DTG curve.

\subsection{Product distribution}

Table 4 shows the distribution of torrefaction products. The torrefaction products of the raw material show bio-oil content is only $29.67 \%$ and the gas content is $40.70 \%$. The bio-oil content from zz80ls is the highest, about 37.34\%, but the gas content is only 28.84\%. Compared with raw materials, the bio-oil content is slight increased by about 2\%. It is possible that after mechanical grinding, the particle size of the raw materials decreases and the surface area increases, which are beneficial for heat and mass transfer, promoting torrefaction of samples and reducing bio-char content. Washing pre-treatment can eliminate some water-soluble metals and other soluble impurities. Pore structures in the raw materials will be changed, which is beneficial in precipitation of volatile components. As a result, bio-oil content increases by $7 \%$ from the sample of zzsx. Ammonia water mainly destroys the structure of lignin, reducing lignin and carbon contents. Such reduction of lignin and carbon contents limits bio-char content (24.26\%) but increases bio-oil content (39.94\%). Compared with ammonia water pre-treatment, ultrasonic ammonia water pre-treatment results in higher bio-char content and lower bio-gas content, which is caused by the high cellulose and hemicellulose contents of ammonia water. Pre-treatment with hydrothermal carbon significantly reduces the hemicellulose content, which is disadvantageous for gas production.

The particle sizes of samples with sulfuric acid pre-treatments significantly influence the distribution of torrefaction products. That is, with decreasing particle size of the samples, the bio-char and bio-oil increase first and then decrease, whereas the amounts of gas components decrease first and then increase. And the bio-char content is lowest (23.81\%) from zz200ls which is with the lowest particle size. Especially, the bio-char (33.82\%) and bio-oil (37.84\%) content is highest for zz80ls 
among all samples. The results is consistent with that the 80 -mesh pre-treatment samples with the lowest hemicellulose content and highest lignin content, indicating that the torrefaction results on the distribution of tristate products are reasonable.

\subsection{Gas component analysis}

Table 5 shows the composition of bio-gas from torrefaction gas. The gaseous components mainly include $\mathrm{CO}_{2}, \mathrm{CO}$, and $\mathrm{CH}_{4}$ and low amounts of $\mathrm{H}_{2}, \mathrm{C}_{2} \mathrm{H}_{4}$, and $\mathrm{C}_{2} \mathrm{H}_{6}$. After washing pre-treatment, $\mathrm{H}_{2}$ content decreases but $\mathrm{CO}_{2}$ content significantly increases from $66.75 \%$ to $74.05 \%$. After ammonia water pre-treatment, $\mathrm{CO}_{2}$ content decreases but CO content increases by 7\%. Especially, the CH4 content increased from $0.55 \%$ to $24.64 \%$. Meanwhile, the heat value of gas increases from 1038 to 3581 $\mathrm{Kcal} / \mathrm{Nm}^{3}$. Ultrasonic pre-treatment with ammonia water slightly influences the gas components. Ultrasonic combined with sulfuric acid pre-treatment is beneficial for acetylene production.

The 30-mesh sulfuric acid pre-treatment will not influence the gas components. However, for the zz80ls samples, hydrogen, CO, and acetylene contents increased. Especially, the $\mathrm{H}_{2}$ and $\mathrm{CO}$ content increased from $1.26 \%$ to $4.47 \%$. It is possible that the cellulose crystal structure is destroyed by the sharp reduction in hemicellulose content. For zz200ls sample, $\mathrm{CO}_{2}$ content further decreases, whereas $\mathrm{CO}$ and $\mathrm{CH}_{4}$ contents greatly increase to $36.13 \%$ and $20.96 \%$, respectively. It is possible that the pore structures and surface area increase, which facilitates methane production. Compared with raw materials, the heat value of bio-gas from zz30ls, zz80ls and zzsr decreased from $3288.47 \mathrm{Kcal} / \mathrm{Nm}^{3}$ to $1615.43 \mathrm{Kcal} / \mathrm{Nm}^{3}$, $1642.87 \mathrm{Kcal} / \mathrm{Nm}^{3}$ and $1411.83 \mathrm{Kcal} / \mathrm{Nm}^{3}$. It is consistent that the hemicellulose content in those samples also decreased. It is possible that those pre-treatment removes the quantities of hemicellulose (HC) which contributes to the heat value of the bio-gas, causing the decrease of heat value. Hydrothermal pre-treatment can influence the growth of CO content. Moreover, the ball-milling pre-treatment reduces the $\mathrm{CO}_{2}$ content. Moreover, the heat value of zzas $^{2 n d}$, zz2001s ${ }^{2 n d}$ is much higher than raw materials, indicating that ammonia water and sulfuric acid with 200mesh pretreatment facilitate the improving of bio-gas energy.

\subsection{Analysis of the physical properties of bio-char}

Table 6 shows physical and chemical properties of bio-char from torrefaction. . 
The results show that the ash content in the sample zz200ls pretreated with sulfuric acid and zzqm pretreated with mechanical milling is $7.94 \%$ and $8.56 \%$, respectively, which is much higher than other pretreated samples. Moreover, the volatile matter content in zz80ls (46.51\%) is much higher than other samples, which was caused by the lignin content in zz80ls. The result of heat value analysis demonstrates that pretreatment has a slightly influence on the calorific value of bio-char. By contrast, the heat value from zzsr after hydrothermal pre-treatment greatly increased from 26.95 to $28.24 \% \mathrm{MJ} / \mathrm{kg}$.

\subsection{Fourier transform infrared (FT-IR) analysis of carbon}

The FT-IR spectra of bio-char from the torrefaction of samples was supplied in the supplementary materials. All samples display a wide peak at $3600-3200 \mathrm{~cm}^{-1}$, which is the stretching vibration peak of -OH in hexagon and hydroxyl in bound water, weakening the peak intensity (Barrosobogeat et al., 2016). Some of these -OH possibly come from carbohydrates in organic matters contained in the pretreated samples and some of them possibly came from water absorbed by the samples. The bio-char from the sample treated by 80-mesh sulfuric acid has a sharp peak, indicating that it contains a considerable amount of free hydroxyls. Moreover, absorption peaks were observed at 3028-2775 $\mathrm{cm}^{-1}$. The peak between 2960 and $2920 \mathrm{~cm}^{-1}$ is the stretching vibration peak of the saturated C-H, which is a saturated fatty carbon (Quan, et al., 2011). The amount of C-H bonds of the carbon of the raw materials pre-treated with sulfuric acid increases, whereas that in $\mathrm{C}-\mathrm{H}$ bonds of the carbon of the raw materials pre-treated with ammonia water and hydrothermal method decreases. The peak at $1705 \mathrm{~cm}^{-1}$ is the stretching vibration absorption peak of $\mathrm{C}=\mathrm{O}$ and serves as the carbonyl structure for ketones and aldehydes. Pre-treatment can weaken the $\mathrm{C}=\mathrm{O}$ bonds, and this phenomenon is beneficial in reducing oxygen content of torrefaction bio-char (Peng et. al., 2016). The region close to $1603 \mathrm{~cm}^{-1}$ is the stretching vibration region of the C-C bonds. The amount of aromatic hydrocarbons in the samples pre-treated with sulfuric acid increases. After the particle size has decreased, the absorption peak intensity increases and the carbon content further increases. The region close to $1210 \mathrm{~cm}^{-1}$ is the stretching vibration region of $\mathrm{C}-\mathrm{O}$, which contains 
substances such as fatty ethers (Chen et al., 2016). Sulfuric acid pre-treatment increases the amount of fatty ethers. The stretching vibration region of C-O at 1105 $\mathrm{cm}^{-1}$ demonstrates the possible existence of molecular structures containing pyrane substances or secondary hydroxyls. Sulfuric acid pre-treatment can increase the amounts of these materials. The absorption peaks close to 816 and $812 \mathrm{~cm}^{-1}$ are contributed by C-H rocking vibration (Schwanninger et al., 2004).

\section{Conclusions and discussions}

1. Hydrothermal pre-treatment can effectively eliminate ash content, while it can increase the concentration of organic volatile components and the amount of fixed bio-char. The calorific value is higher in hydrothermal pre-treatment than in other pre-treatments. The decreasing order of the deashing capabilities of the different pre-treatments is as follows: zzsr $>$ zzcls $>$ zzsx $>$ zz30ls $>$ zzas $>$ zzcas $>$ zz80ls $>$ zz200ls > zzqm.

2. After ammonia water pre-treatment, cellulose content increases greatly, whereas hemicellulose and lignin contents decrease due to their dissolution under alkaline conditions. Ultrasonic pre-treatment with ammonia water can facilitate dissolution of lignin and this pretreatment displays a certain selectivity in terms of the dissolution of the three principal components. The ultrasonic sulfuric acid pre-treatment slightly influences the three principal components. Hydrothermal pre-treatment can dissolve hemicellulose, thereby reducing hemicellulose content. Ball-milling pre-treatment, which destroys the component structures of the raw materials, can mechanically dissolve more materials under the effect of acid detergent, thereby increasing the hemicellulose content but reducing the destroyed cellulose and lignin contents.

After sulfuric acid pre-treatment for 30-mesh raw materials, hemicellulose content drops significantly, indicating that hemicellulose is easily dissolved under this condition. On the contrary, cellulose and lignin contents increase. After sulfuric acid pre-treatment for 80-mesh raw materials, with the decrease in particle size and increase in contact area between raw materials and sulfuric acid, hemicellulose dissolution rate increases significantly while the cellulose dissolution rate further increases. After sulfuric acid pre-treatment for 200-mesh raw materials, which further reduces the particle size of raw materials, the hemicellulose structure is destroyed by 
sulfuric acid. The destroyed hemicellulose more easily reacts with cellulose, greatly reducing the cellulose content and sulfuric acid. As a result, the dissolution of hemicellulose and cellulose decreases or stops, resulting in the increase in hemicellulose content. .

3. Before and after pre-treatments, the initial reaction temperature of the torrefaction of the raw materials changes between $257^{\circ} \mathrm{C}$ and $332{ }^{\circ} \mathrm{C}$, and the increasing order of the initial temperature is as follows: zzqm $<$ zzyl $<$ zzcls $<$ zzcas $<$ zz30ls < zzas < zzsr; the ending temperature changes between $349{ }^{\circ} \mathrm{C}$ and $381^{\circ} \mathrm{C}$, and the increasing order of the ending temperature is as follows: zzqm $<$ zzyl $<$ zzas $<$ zzcls $<$ zz30ls $<$ zzcas $<$ zzsr. With the increase in torrefaction reaction temperature, mass loss increases. When the maximum reaction rate, that is, the maximum mass loss, was reached, the temperature changes significantly ranging between $321{ }^{\circ} \mathrm{C}$ and $365^{\circ} \mathrm{C}$, and the increasing order of the changes in temperature is as follows: zzqm $<$ zzcls $<$ zzyl $<$ zz30ls $<$ zzcas $<$ zzas $<$ zzsr. The mass loss at the maximum reaction rate fluctuates at $8.6-15.7 \mathrm{wt} \% / \mathrm{min}$, and the decreasing order of mass loss is as follows: zzsr $>$ zzas $>$ zz30ls $>$ zzcas $>$ zzcls $>$ zzqm $>$ zzyl. This result demonstrates that different pre-treatments will influence to a certain extent the torrefaction behaviors of biomass.

4. After pre-treatments, the bio-oil contents of torrefaction products increase continuously. Ball-milling pre-treatment increases bio-oil content of the torrefaction products but reduces the bio-char content. Pre-treatment with water dissolves water-soluble metals and other soluble impurities in water. Pore structures in the raw materials increase, which is beneficial for the precipitation of volatile components, thereby increasing bio-oil content. Ammonia water mainly destroys the structure of lignin, reducing lignin and bio-char contents. Such reduction of lignin and bio-char contents limits bio-char production but increases bio-oil content. Compared with the ultrasonic ammonia water pre-treatment, ammonia water pre-treatment has higher bio-oil output but results in lower bio-char content, and this finding is caused by the high cellulose and hemicellulose contents of ammonia water; however, this treatment results in low lignin content. Different sizes of the raw material after sulfuric acid pre-treatments influence the tristate products of torrefaction. The influencing law is as follows: with the reduction in particle size of the raw material, the bio-char and bio-oil outputs increase first and then decrease, whereas the amounts of gas components decrease first and then increase. Based on the analysis results of three principal 
components, the 80-mesh pre-treatment results in the lowest hemicellulose content and highest lignin content, indicating that the torrefaction results on distribution of tristate products are reasonable. The hydrothermal pre-treatment significantly reduces hemicellulose content, which is disadvantageous for gas production.

5. Pre-treatment influences bio-chars of the torrefaction. Specifically, the amount of C-H bonds in bio-char of samples pre-treated with sulfuric acid increases but that in bio-char of samples pre-treated with ammonia water and hydrothermal method decreases. All pre-treatments weaken $\mathrm{C}=\mathrm{O}$ bonds, which is beneficial for reduction of oxygen content in torrefaction bio-char. The amount of aromatic hydrocarbons in samples pretreated with sulfuric acid increases. After particle size has decreased, the absorption peak intensity increases. Additionally, sulfuric acid pre-treatment increases the amount of fatty ethers. The stretching vibration region of C-O at $1105 \mathrm{~cm}^{-1}$ demonstrates the possible existence of molecular structures containing pyrane substances or secondary hydroxyls. Sulfuric acid pre-treatment can increase the amounts of these materials.

\section{Acknowledgement}

Supported by the Science and Technology Planning Project of Guangdong Province, China (Grant No.2016A020210073), the National Science Foundation of China (Grant NO.51576071), Key Laboratory of Biomass Energy and Material(KLBEM), (Grant NO.JSBEM201706) and the Science and Technology Planning Project of Guangdong Province, China (Grant No.2015B020237010).

\section{Reference}

[1]Chen, X., Li, H., Sun, S., Cao, X., \& Sun, R. (2016). Effect of hydrothermal pretreatment on the structural changes of alkaline ethanol lignin from wheat straw. Sci. Rep., 6, 39354-.

[2]Aid, T., Hyvärinen, S., Vaher, M., Koel, M., \& Mikkola, J. P. (2016).

Saccharification of lignocellulosic biomasses via ionic liquid pretreatment. Ind. Crops Prod., 92, 336-341.

[3]Carvalho, D. M. D., Queiroz, J. H. D., \& Colodette, J. L. (2016). Assessment of 
alkaline pretreatment for the production of bioethanol from eucalyptus, sugarcane bagasse and sugarcane straw. Ind. Crops Prod., 94, 932-941.

[4]Si, S., Chen, Y., Fan, C., Hu, H., Li, Y., \& Huang, J. (2015). Lignin extraction distinctively enhances biomass enzymatic saccharification in hemicelluloses-rich miscanthus species under various alkali and acid pretreatments. Bioresour. Technol., 183C, 248-254.

[5]Agbor, V. B., Cicek, N., Sparling, R., Berlin, A., \& Levin, D. B. (2011). Biomass pretreatment: fundamentals toward application. Biotechnol. Adv., 29, 675-685. [6]Sindhu R, Binod P, Mathew AK, et al. (2017). Development of a novel ultrasound-assisted alkali pretreatment strategy for the production of bioethanol and xylanases from chili post harvest residue. Bioresour. Technol., In press.

[7]Karray, R., Hamza, M., \& Sayadi, S. (2015). Evaluation of ultrasonic, acid, thermo-alkaline and enzymatic pre-treatments on anaerobic digestion of ulva rigida, for biogas production. Bioresour. Technol., 187, 205-213.

[8]Lu, H., Lv, C., Zhang, M., Liu, S., Liu, J., \& Lian, F. (2017). Optimization of hydrothermal pretreatment for co-utilization c-5 and c-6 sugars of cassava alcohol residue. Energy Conversion \& Management, 132, 251-260.

[9]Woytiuk, K., Campbell, W., Gerspacher, R., Evitts, R. W., Phoenix, A., \& Kalogirou, S. A., et al. (2017). The effect of torrefaction on syngas quality metrics from fluidized bed gasification of src willow. Renew. Energy.101, 409-416. [10]Doddapaneni, T. R. K. C., Konttinen, J., Hukka, T. I., \& Moilanen, A. (2016). Influence of torrefaction pretreatment on the pyrolysis of eucalyptus clone: a study on kinetics, reaction mechanism and heat flow. Ind. Crops Prod., 92, 244-254. [11]Wigley, T., Yip, A. C. K., \& Pang, S. (2016). Pretreating biomass via demineralisation and torrefaction to improve the quality of crude pyrolysis oil. Energy, 109, 481-494.

[12]Chen, D., Li, Y., Deng, M., Wang, J., Chen, M., \& Yan, B., et al. (2016). Effect of torrefaction pretreatment and catalytic pyrolysis on the pyrolysis poly-generation of pine wood. Bioresour. Technol., 214, 615-622.

[13]Tran, K. Q., Bach, Q. V., Trinh, T. T., \& Seisenbaeva, G. (2014). Non-isothermal 
pyrolysis of torrefied stump-a comparative kinetic evaluation. Appl. Energy, 136, 759-766.

[14] Basu, P., Sadhukhan, A. K., Gupta, P., Rao, S., Dhungana, A., \& Acharya, B. (2014). An experimental and theoretical investigation on torrefaction of a large wet wood particle. Bioresour. Technol., 159, 215-222.

[15]Chen, W. H., Huang, M. Y., Chang, J. S., \& Chen, C. Y. (2015). Torrefaction operation and optimization of microalga residue for energy densification and utilization. Appl. Energy, 154, 622-630.

[16]Zhang, S. (2016). Physicochemical properties and combustion behavior of duckweed during wet torrefaction. Bioresour. Technol., 218, 1157-1162.

[17]Zhang, X., Zhang, S., Yang, H., Shao, J., Chen, Y., \& Feng, Y., et al. (2015).

Effects of hydrofluoric acid pre-deashing of rice husk on physicochemical properties and co 2, adsorption performance of nitrogen-enriched biochar. Energy, 91, 903-910. [18]He, Z., Wang, Z., Zhao, Z., Yi, S., Mu, J., \& Wang, X. (2017). Influence of ultrasound pretreatment on wood physiochemical structure. Ultrason. Sonochem., 34, 136-141.

[19]Barrosobogeat, A., Alexandrefranco, M., Fernándezgonzález, C., Macíasgarcía, A., \& Gómezserrano, V. (2016). Preparation of activated carbon- $\mathrm{SnO}_{2}, \mathrm{TiO}_{2}$, and $\mathrm{WO}_{3}$ catalysts study by FT-IR spectroscopy. Ind. Eng. Chem. Res., 55, 5200-5206 [20]Quan, G. X., Yan, J. L., \& Ding, C. (2011). Preparation and characterization of biomass carbon adsorbent from rice husk and its adsorption properties on p-chlorophenol. Adv. Mater. Res., 356-360, 510-513.

[21]Peng, W., Ge, S., Liu, Z., \& Furuta, Y. (2016). Adsorption characteristics of sulfur powder by bamboo charcoal to restrain sulfur allergies. Saudi J. Biol. Sci., 24, 103-107.

[22]Chen, T., Liu, R., \& Scott, N. R. (2016). Characterization of energy carriers obtained from the pyrolysis of white ash, switchgrass and corn stover-biochar, syngas and bio-oil. Fuel Process. Tech., 142, 124-134.

[23]Schwanninger, M., Rodrigues, J. C., Pereira, H., \& Hinterstoisser, B. (2004). 
Effects of short-time vibratory ball milling on the shape of FT-IR spectra of wood and cellulose. Vib. Spectrosc., 36, 23-40. 


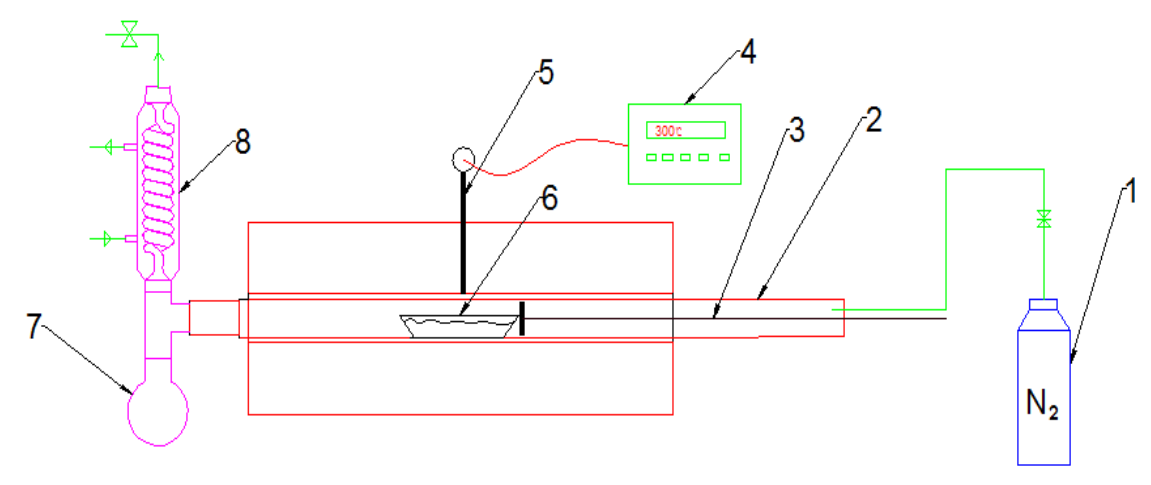

1 - nitrogen cylinder; 2 - high-temperature resistance quartz tube; 3 - push rod; 4 programming temperature controller; 5 - thermocouple; 6 - railboat; 7 -distilling flask; 8 - S-shaped condenser pipe; and 9 - tube-type resistance furnace

Fig. 1 Equipment used in sample torrefaction



Fig. 2 Results of TG curve analysis of Phyllostachys edulis 


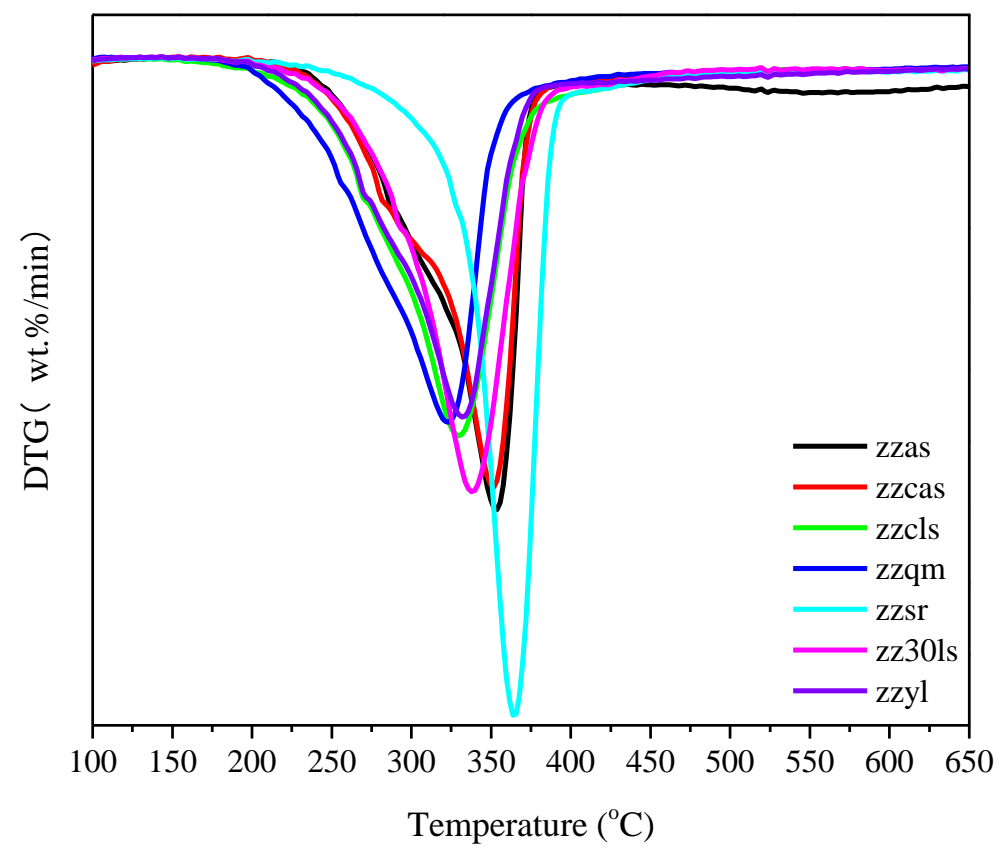

Fig. 3 Results of DTG curve analysis of Phyllostachys edulis 
Table 1 Chemical and physical properties of pretreated samples

\begin{tabular}{llllll}
\hline Samples & $\begin{array}{l}\text { Moisture } \\
\text { content }(\%)\end{array}$ & $\begin{array}{l}\text { Ash } \\
\text { content } \\
(\%)\end{array}$ & $\begin{array}{l}\text { Volatile } \\
\text { matter }(\%)\end{array}$ & $\begin{array}{l}\text { Fixed } \\
\text { carbon } \\
(\%)\end{array}$ & $\begin{array}{l}\text { Heat value } \\
(\mathrm{MJ} / \mathrm{kg})\end{array}$ \\
\hline zzyl & 5.64 & 1.78 & 75.85 & 16.91 & 17.65 \\
zzsx & 9.49 & 0.57 & 75.98 & 13.96 & 17.94 \\
zzas & 6.31 & 0.73 & 79.27 & 13.67 & 17.65 \\
zzcas & 10.27 & 0.74 & 74.87 & 14.12 & 17.46 \\
zzcls & 7.01 & 0.52 & 75.74 & 16.73 & 17.79 \\
zz301s & 7.7 & 0.67 & 74.99 & 16.64 & 17.94 \\
zz801s & 7.29 & 1.19 & 77.16 & 14.36 & 17.45 \\
zz2001s & 9.33 & 1.75 & 75.46 & 16.73 & 17.35 \\
zzsr & 5.51 & 0.35 & 77.15 & 16.99 & 19.35 \\
zzqm & 7.63 & 2.26 & 73.81 & 16.30 & 18.91 \\
\hline
\end{tabular}

Table 2 The principal components of pretreated samples

\begin{tabular}{lllll}
\hline Samples & $\begin{array}{l}\text { Hemicellulose } \\
(\%)\end{array}$ & $\begin{array}{l}\text { Cellulose } \\
(\%)\end{array}$ & Lignin $(\%)$ & $\begin{array}{l}\text { Mass loss } \\
(\%)\end{array}$ \\
\hline zzyl & 18.66 & 51.55 & 20.44 & 90.65 \\
zzsx & 19.77 & 52.87 & 21.76 & 94.4 \\
zzas & 17.80 & 58.37 & 19.96 & 96.13 \\
zzcas & 21.02 & 53.88 & 20.66 & 95.56 \\
zzcls & 19.46 & 53.11 & 21.97 & 94.54 \\
zz301s & 12.47 & 57.91 & 23.45 & 93.83 \\
zz801s & 13.51 & 57.51 & 28.94 & 89.96 \\
zz2001s & 10.06 & 54.91 & 26.80 & 91.77 \\
zzsr & 8.69 & 56.93 & 22.64 & 88.26 \\
zzqm & 20.13 & 47.43 & 19.61 & 87.17 \\
\hline
\end{tabular}

Table 3 TG analysis of the pretreated samples

\begin{tabular}{lllll}
\hline \multirow{2}{*}{ Samples } & $\begin{array}{l}\text { Temperature zone of } \\
\text { mass loss }\left({ }^{\circ} \mathrm{C}\right)\end{array}$ & $\begin{array}{l}\text { Temperature } \\
\text { of Peak } /{ }^{\circ} \mathrm{C}\end{array}$ & $\begin{array}{l}\text { Mass loss } \\
(\%)\end{array}$ & $\begin{array}{l}\text { Mass loss rate } \\
(\mathrm{wt} \% / \mathrm{min})\end{array}$ \\
\hline zzyl & $268.05-360.64$ & 332.05 & 60.78 & -8.67 \\
zzas & $293.56-362.98$ & 353.38 & 61.90 & -10.79 \\
zzcas & $285.55-367.89$ & 349.97 & 62.13 & -10.25 \\
zzcls & $271.47-364.05$ & 329.49 & 66.84 & -9.04 \\
zz301s & $289.39-364.05$ & 339.70 & 68.82 & -10.38 \\
zzsr & $332.05-381.97$ & 365.70 & 64.42 & -15.73 \\
zzqm & $257.39-349.97$ & 321.70 & 64.37 & -8.73 \\
\hline
\end{tabular}


Table 4 Distribution of torrefaction products

\begin{tabular}{lccc}
\hline Samples & Bio-char $(\%)$ & Bio-oil $(\%)$ & Bio-gas $(\%)$ \\
\hline zzyl & 29.62 & 29.67 & 40.70 \\
zzsx & 34.25 & 36.52 & 29.25 \\
zzas & 24.26 & 35.80 & 39.94 \\
zzcas & 29.16 & 35.07 & 33.04 \\
zzcls & 30.50 & 34.16 & 35.34 \\
zz301s & 32.49 & 35.85 & 31.66 \\
zz801s & 33.82 & 37.34 & 28.84 \\
zz2001s & 23.81 & 37.22 & 38.98 \\
zzsr & 31.87 & 34.41 & 33.71 \\
zzqm & 28.34 & 31.37 & 40.29 \\
\hline
\end{tabular}

Table 5 Composition and heat value of bio-gas from torrefaction

\begin{tabular}{lcccccccc}
\hline Sample & \multicolumn{7}{c}{ Bio-gas composition (\%) } & Heat value \\
\cline { 2 - 6 } & $\mathrm{H}_{2}$ & $\mathrm{CO}$ & $\mathrm{CH}_{4}$ & $\mathrm{CO}_{2}$ & $\mathrm{C}_{2} \mathrm{H}_{4}$ & $\mathrm{C}_{2} \mathrm{H}_{6}$ & $\left(\mathrm{Kcal} / \mathrm{Nm}^{3}\right)$ \\
\hline zzyl $^{1 \text { th }}$ & 1.26 & 31.45 & 0.55 & 66.75 & - & - & 1038.89 \\
zzyl $^{2 \text { nd }}$ & 1.63 & 30.99 & 6.69 & 56.66 & 0.73 & 3.09 & 2249.58 \\
zzsx $^{1 \text { th }}$ & 0.18 & 24.91 & 0.87 & 74.05 & - & - & 839.25 \\
zzsx $^{2 \text { nd }}$ & 0.63 & 36.50 & 1.35 & 60.92 & 0.60 & - & 1338.92 \\
zzas $^{1 \text { th }}$ & 0.15 & 38.79 & 0.68 & 60.38 & - & - & 1238.75 \\
zzas $^{2 \text { nd }}$ & 1.81 & 38.63 & 24.64 & 34.80 & 0.12 & - & 3581.25 \\
zzcas $^{1 \text { th }}$ & 1.23 & 31.94 & 0.98 & 65.85 & - & - & 1093.64 \\
zzcas $^{2 \text { nd }}$ & 0.41 & 42.63 & 1.93 & 54.47 & 0.92 & - & 1620.67 \\
zz301s $^{1 \text { th }}$ & 1.75 & 31.77 & 5.51 & 60.45 & 0.53 & - & 1615.43 \\
zz801s $^{1 \text { th }}$ & 4.47 & 34.94 & 1.04 & 57.19 & 2.34 & - & 1642.87 \\
zz2001s $^{1 \text { th }}$ & 1.63 & 24.13 & 0.49 & 73.38 & 0.36 & - & 878.28 \\
zz2001s $^{2 \text { nd }}$ & 1.08 & 36.13 & 20.96 & 40.77 & 1.07 & - & 3277.56 \\
zzsr $^{1 \text { th }}$ & 1.23 & 35.45 & 2.13 & 60.52 & 0.68 & - & 1411.83 \\
zzqm $^{1 \text { 1th }}$ & 0.31 & 35.69 & 0.36 & 63.65 & - & - & 1119.73 \\
zzqm $^{2 \text { 2nd }}$ & 1.99 & 32.53 & 13.46 & 48.19 & 0.52 & 3.30 & 2954.25 \\
\hline
\end{tabular}


Table 6 Physical and chemical properties of bio-char from torrefaction of pretreated samples

\begin{tabular}{llllll}
\hline Samples & $\begin{array}{l}\text { Moisture } \\
\text { content }(\%)\end{array}$ & $\begin{array}{l}\text { Ash content } \\
(\%)\end{array}$ & $\begin{array}{l}\text { Volatile } \\
\text { matter }(\%)\end{array}$ & $\begin{array}{l}\text { Fixed } \\
\text { carbon }(\%)\end{array}$ & $\begin{array}{l}\text { Heat value } \\
(\mathrm{MJ} / \mathrm{kg})\end{array}$ \\
\hline zzyl & 3.21 & 5.00 & 30.15 & 61.64 & 26.95 \\
zzsx & 1.73 & 2.78 & 37.69 & 57.80 & 27.72 \\
zzas & 3.19 & 4.74 & 26.17 & 65.90 & 27.52 \\
zzcas & 2.75 & 2.69 & 35.47 & 59.09 & 27.81 \\
zzcls & 1.65 & 2.86 & 34.30 & 61.19 & 27.22 \\
zz301s & 2.27 & 2.06 & 37.36 & 58.31 & 26.75 \\
zz801s & 1.27 & 3.05 & 46.51 & 49.17 & 26.65 \\
zz2001s & 3.43 & 7.94 & 23.33 & 65.30 & 26.68 \\
zzsr & 1.83 & 1.78 & 34.59 & 61.80 & 28.24 \\
zzqm & 2.82 & 8.56 & 26.51 & 62.11 & 26.15 \\
\hline
\end{tabular}

